\title{
Design of an Automated Secure Garage System Using License Plate Recognition Technique
}

\author{
Afaz Uddin Ahmed, Taufiq Mahmud Masum , Mohammad Mahbubur Rahman \\ Dept. of Electrical \& Electronic Engineering, Chittagong University of Engineering and Technology, Chittagong, \\ Bangladesh \\ E-mail:afazbd@gmail.com;masum_liby@yahoo.com;mahbub.rahman336@gmail.com
}

\begin{abstract}
Modern technologies have reached our garage to secure the cars and entrance to the residences for the demand of high security and automated infrastructure. The concept of intelligent secure garage systems in modern transport management system is a remarkable example of the computer interfaced controlling devices. License Plate Recognition (LPR) process is one of the key elements of modern intelligent garage security setups. This paper presents a design of an automated secure garage system featuring LPR process. A study of templates matching approach by using Optical Character Recognition (OCR) is implemented to carry out the LPR method. We also developed a prototype design of the secured garage system to verify the application for local use. The system allows only a predefined enlisted cars or vehicles to enter the garage while blocking the others along with a central-alarm feature. Moreover, the system maintains an update database of the cars that has left and entered into the garage within a particular duration. The vehicle is distinguished by the system mainly based on their registration number in the license plates. The tactics are tried on several samples of license plate's image in both indoor and outdoorsetting.
\end{abstract}

Index Terms - Automatic Secure Garage, LPR, Matlab Interfacing, Matlab Image Processing, OCR, Template Matching Technique

\section{Introduction}

All the essential daily equipment in our life is becoming automatic. This saves a lot of time and energy as well. Garage doors are also becoming automatic. The security system of garage-doors is one of the important is sue in our offices and residences. The security problem of a garage has been investigated for the last decade [1]. Many researchers have proposed various methods of security systems [2,3]. Each of these systems has its own advantages and disadvantages [4]. Most of the modern garages have security systems, which detect vehicles authentication while entering and exiting [5]. However, these security systems often prevent accommodating equipment and some are unattractive solution for the existing system. It is clear that efficient garage security system today involves interdisciplinary research areas. In modern security system, the image processing, microcontroller and sensor technologies play an important role [6]. Considering the fact, the objective of the work was to develop a security system for an automatic garage door. The security system has a way of identifying a car and, if the car is authorized to enter the area, the door opens and lets the car to move in. The garage door automatically closes as the car passes through. The door also operates automatically when car moves out-off the garage. This mechanism is done by using LPR Technique. There are many LPR applications in recent days, such as- automatic highway toll collection, vehicle theft prevention, enforcement of traffic rules and so on. In this paper, we focused on LPR method interfacing with an automated garage system. A prototype design is also provided with performance evaluation. Rest of the paper is arranged as follows, Methodology in section II, Overview of the mechanical part in section III, Image sensing and matching in section IV, Design of the interfacing circuit in section V, Performance analysis in section VI, Features and applications in section VII and Conclusion in VIII.

\section{Methodol ogy}

Now a days, we see intelligent garage systems with car-maneuvers operated with sensor-fuzzy logic controls and spring layer lifted intelligent garage [7, 8]. Automatic parking control system with lifting and transferring stereo garage by using IPC and PLC are being implemented in some parts of the first world countries. [9]. Some of these stereo garage devices are linked up with network to share information over a fair distance to control the mechanism and secure the operation remotely [10]. Some intelligent parking design and transport management system has been proposed in [11-13]. In this paper, we focused on the car's LPR technique to operate an automated secure garage system. Generally, the LPR system consists of four major modules: image sensing, LPR, seg mentation and recognition of specific characters (LPR). A local server contains the list of the cars that are allowed to get in the garage. This database can be edit by any of the house owners or the authority in charge. As soon as the 
car appears in the front gate, it will activate a sensor which will be liked with a central control system (here we used a pc with MATLAB). The system grabs a picture of the license plate and analyses the reference cars' license plate number. After a successful match, the garage opens automatically allowing the car to get in. While passing the garage, the car again activates a sensor that executes the closing process of the garage doors. The system informs the car owner about the entrance of the car in the garage. The same process repeats when the car wants to get out of the garage. The LPR process is done by gray-image templates matching using optical character recognition process (OCR). The interfacing with the automated garage system is done by simple control circuits with stepper motor that gives flexibility to rotate both ways by just changing the phases.

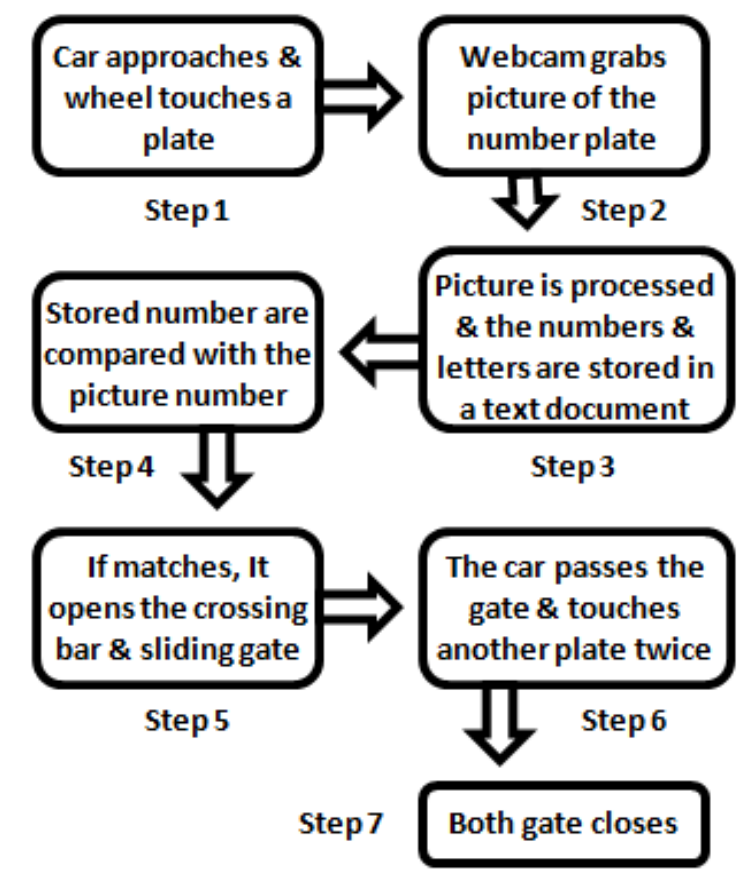

Fig. 1: Systematically process of the system

Step1 The car approaches in front of the garage and the wheel touches a plate. In addition, a signal passes from the plate through the parallel port of a computer.

Step2 Sensing this signal, MATLAB starts the camera and grabs a picture of the car's license plate. The picture is then stored.

Step3 The stored picture is then processed through Optical Character Recognition and then the number and letters are saved in a text document.

Step4 Image form references are taken and then converted into text. Then the both texts are compared. If the image matches, it gives logic 1 to step 5 .

Step5 If step 4 gives logic 1 , then the motor interfaced by PC rotates and the sliding and cross bar gates open.
Step6 When the car passes the gate, it touches another plate twice (for the front wheel and the back wheel). This sends two simultaneous signals to the PC.

Step7 Then the motor rotates back to close both the doors.

\section{Overview of the Mechanical Part}

The mechanical part is a miniature of a gate that is shown in Fig. 2. This gate model is similar to the gate model that we proposed for practical application. The sturcture is made of wood to get the fle xibility of shapes and modification in the workshop. The frame of the gate is constructed on a wooden driveway. The driveway has two sensors at two different places, one before approaching the gate and another just after the gate. As these plates act like weight sensors, springs are attached to the plates to ensure the proper operation of the circuit. The gate has two parts. One crossing bar which is in front of the gate a horizontally siding doors that are next to the crossing bar. With the crossing bar, there is a camera attached for the graphical input of the license plate. The sliding doors have rack attached with it on the top. This rack meshes with a pinion that is coupled with two stepper motors. The crossing bar also has a motor coupled, which is used to move the crossing bar up and down. A box with wheels attached is used as a car.

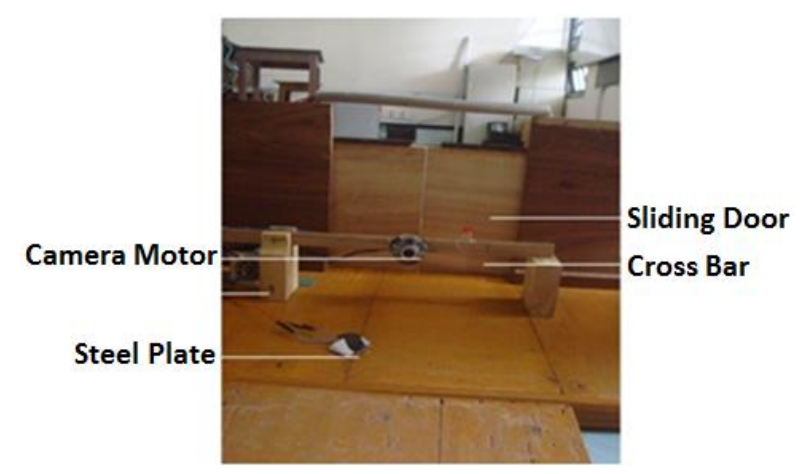

Fig. 2: Wooden model of the gate and the driveway

When a car approaches the first plate, it touches the weight sensor, which closes the input circuit and sends a signal to the computer. The computer turns on the camera and takes the photograph of the license plate of the car. The photo is then filtered and matched with the photos saved in the database (the data is sent electronically), if it matches with any of the cars number in the list, the arrangement of opening the gates executes. Frist the stepper motor for the bar is switched on with a particular number of rotations and the bar moves up. As soon as the moving bar stops, the gate motor gets the signal for opening. The two sliding door moves in the opposite direction making a route for the cars to pass through it. When the car crosses the sliding door, it again touches a weight sensor and closes the circuit (usually the last plate operates after two 
consecutive contacts because of the front tire and the back tire). Than all the gates close by rotating in the opposite direction and gets in the original position.

\section{Image Sensing and Matching}

The image processing and gray level template matching part is the heart of this process. The image is first converted to gray level. Than it is converted to binary format to match with individual characters of the reference picture. Each character is compared with a set of templates that are stored as a reference model. After that the character is been recognized and stored in a text document. Here in this system we used English alphabets and numerical numbers: 36 te mplates $(\mathrm{A}-\mathrm{Z}=$ $26,0-9=10)$ [14]. Changing the set of references, we can change the language of the system. We can also use multiple languages by using more than one set of alphabets and numerical numbers.

Following are the steps how actually the image sensing works -

First, a library for templates is created. This library contains images of different characters in gray format that can appear on the license plate. This is done before any image capturing or processing is done [15].

When an image of the license plate is taken, the image is into grayscale. The digital image is then converted to binary image. The white part corresponds to 1 and the black part to 0 .

The image contains two parts, one with vast black area and other with white numbers on the black background. The number part is separated and appeared in a black area with white backg round. The number part itself has parts equal to the nu mber of characters. These characters in the license plate are compared with reference templates. The matched template indicates particular alphabets and numerical numbers and it is stored in a specific text document (Cars.txt). Now, as all the license plate nu mbers of the allowed cars, which are in the library folder, are checked one by one and matched them with the previous file (Cars.txt). If any of the name matches with the car.txt, the logic will show 1 and the gate will open systematically. Then email notification will be sent to the owners of the car to make inform about the cars status. If none of the reference files matches, the output will be a red light. These processes are done by MATLAB. We have also made a database record for the cars that goes in and gets out with in last 6 hours. We have used a computer with video input device (webcam) and MATLAB programming to detect the optical characters template matching technique. Template matching in digital image processing for recognizing characters is very effective method. It can be used to detect edges in images. Here we used Grey-level template matching.

In matching process, the templates' images are moved to all possible points/scale in the source image to compute the numerical indication on success rate of the template matching in that image. This matching is done in pixel-by-pixel.

\subsection{Optical Character Recognition}

The aim of optical character recognition (OCR) is to identify optical patterns like numerical numbers or letters or symbols in any picture. There are several steps in OCR that includes segmentation, feature extraction, and classification. Each of these steps is a field unto itself [14]. Figure 3 shows different stages of this process.

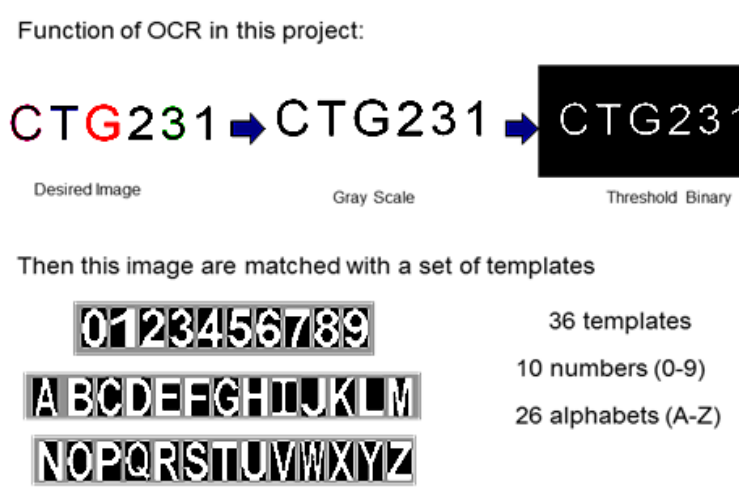

Fig. 3: Different stages of Optical Character Recognition

Binary Image
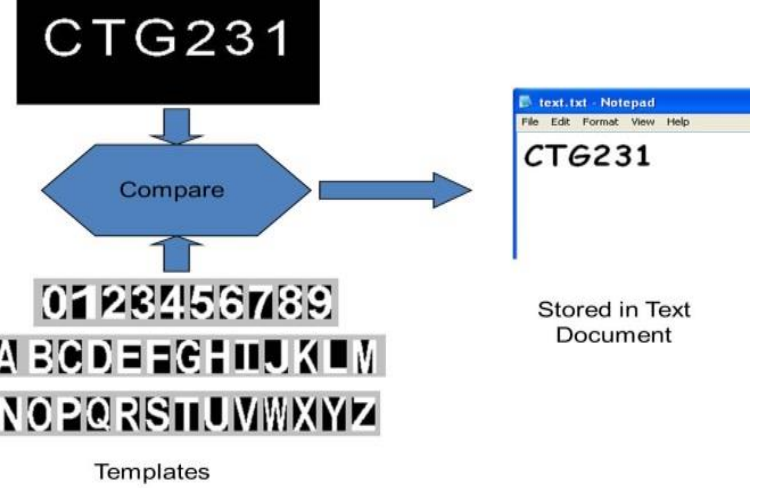

Fig. 4: Comparison and storage of the converted binary image

First, the desired image is converted into grayscale. Then it will be converted in threshold binary. The image will be then compared with a set of alphabet and number image that we stored as templates. The conversion and matching will make an output of successful matching in a text document. Fig. 4 shows the process of storing matched image into a text document.

The algorith $m$ that was followed during the process is briefly described below- 


\subsection{The Classification Process}

Classification is common for any kind of classifier. There are two main steps in developing classifier training and testing. These steps can be split into more sub-steps [6, 16]. Fig. 5 shows the block diagram representation of the sub-steps of this classification process.

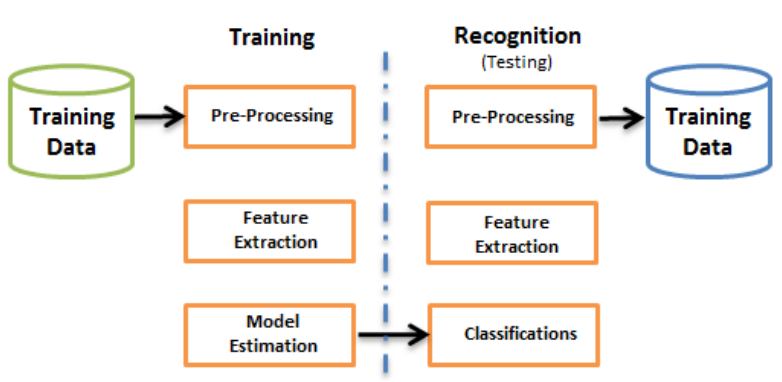

Fig. 5: The pattern classification process of OCR (Pre-processing)

\section{a) Training}

- Pre-processing - Processes the collected data so it is in a suitable form for later processes.

- Feature abstraction - Minimizes the total amount of data by extracting related information, usually results in a vector of scalar values.

- Model Estimation - for each class of the training data, the finite set of feature vectors needs to estimate as a model.

\section{b) Testing}

- Pre-processing-(same as above)

- Feature extraction - (same as above)

- Classification - Differentiate the feature vectors to several models and extract the highest possible match.

These steps are performed in OCR [17].

1. Binarization- It is converting an image of up to 256 gray levels to a black and white image.

2. Morphological Operators - Removes holes and is olated specks from the characters.

3. Segmentation - Confirms connectivity of outlines labels and separate. Using the bwlabel' and 'regionprops' functions of MATLAB, it can done easily [16]. Seg mentation is the most important stage of the pre-processing method. It assists the recognizer to extract features from individual character. In the case of handwritten text, which is more complex, it becomes more difficult as characters are tending to be linked to each other. The overall process of OCR in this project is shown in Figure 6.

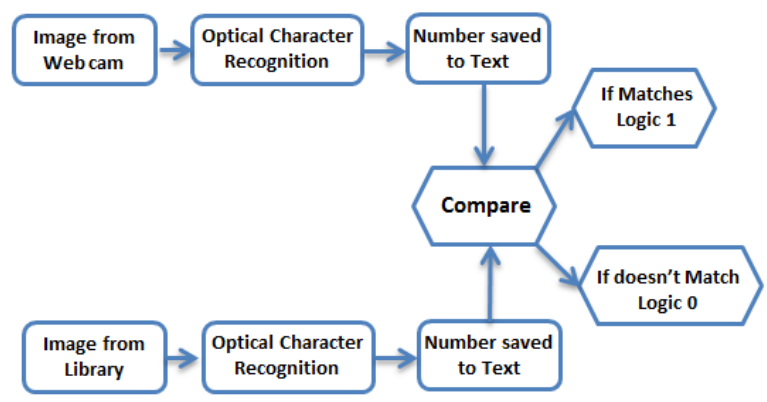

Fig. 6: Block diagram of the program

\section{Design of the Interfacing Circuit}

To design the control setup, we used eight relays, two ULN2003 IC and a $12 \mathrm{~V}$ power supply. The circuit is connected as shown in Fig.7 below. When a signal comes to first pin of the ULN2003, it amplifies the current and relay is switched on. As the relay is closed, the motor gets a pulse to rotate one-step of 1.8 degrees. The next comes to the second pin, and the second relay causes another step rotation. As the ULN pin keeps getting sequential signal, the motor keep rotating [18]. The number of signal required is set by the program and the motors are controlled. The same thing is done for other motors. We used three motors. Two for the sliding gates and one for the crossing bar.

A unipolar stepper motor has two windings per phase, one for each direction of magnetic field. Since in this arrangement a magnetic pole can be reversed without switching the direction of current, the commutation circuit can be made very simple (eg. a single transistor) for each winding. Typically, given a phase, one end of each winding is made common: giving three leads per phase and six leads for a typical two phase motor. Often, these two-phase commons are internally joined, so the motor has only five leads.

Phase shifting of the stepper motor:
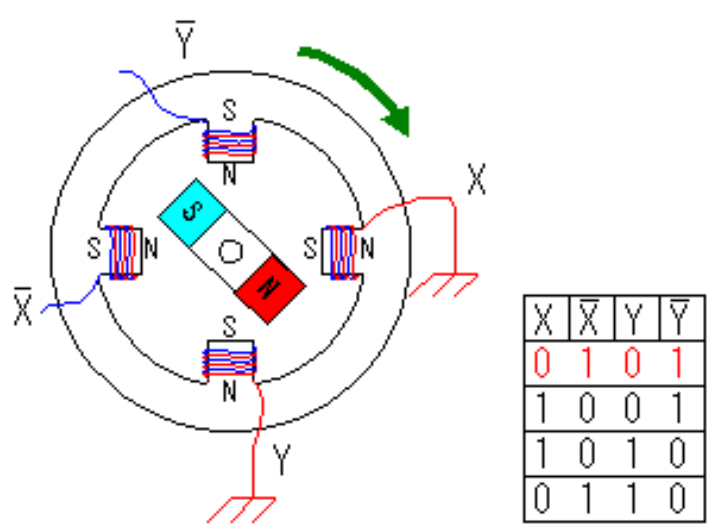

Fig. 7: Clockwise Rotation 


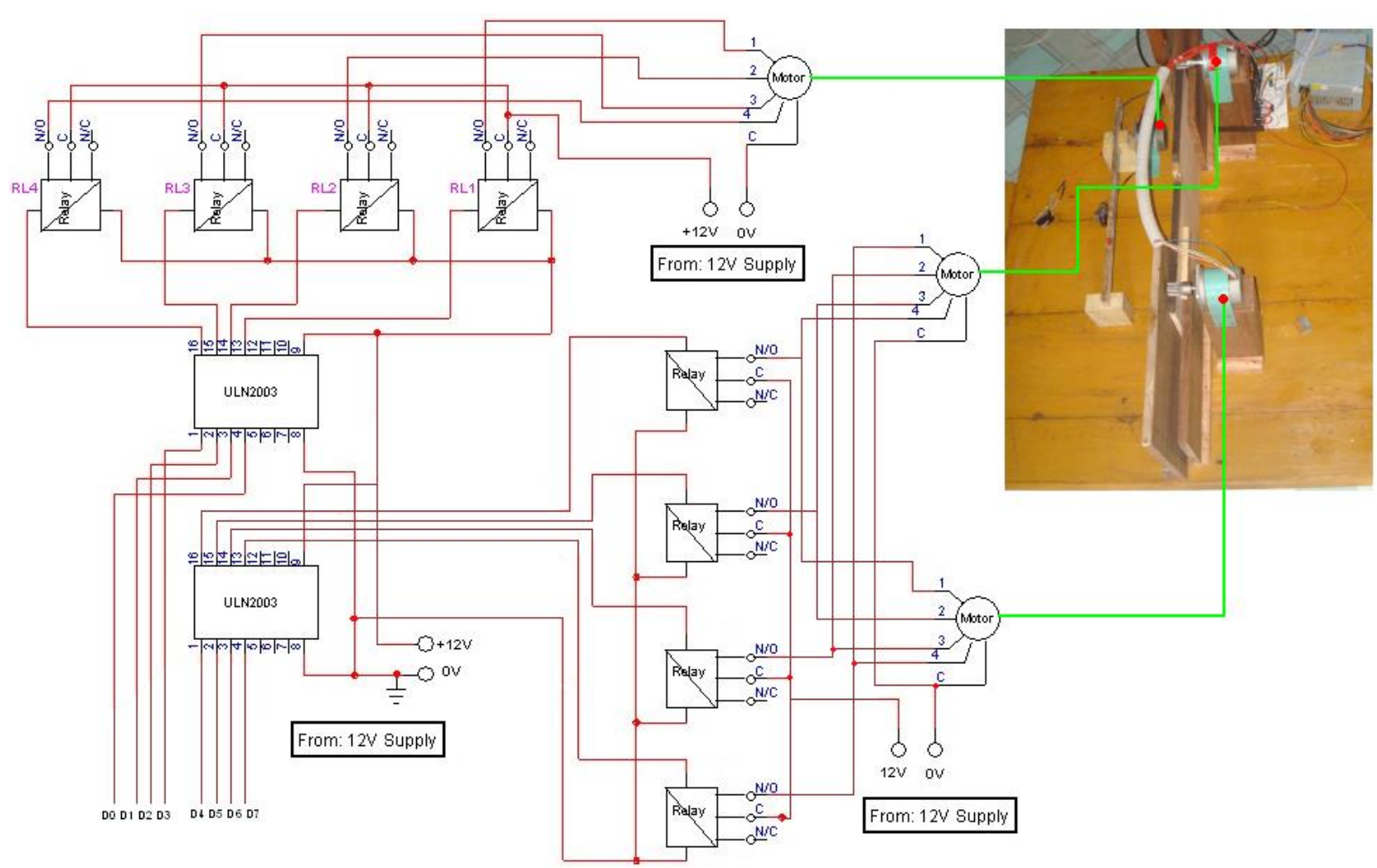

Fig. 8: Connection diagram of the motor with the interfacing circuit

Table 1: Functional Table

$\mathrm{X}, \overline{\mathrm{X}} \mathrm{Y}$ and $\overline{\mathrm{Y}}$ are controlled in the following order.

\begin{tabular}{|c|c|c|c|c|}
\hline$X$ & $\bar{X}$ & $Y$ & $\bar{Y}$ & Step angle \\
\hline 0 & 1 & 0 & 1 & $0^{\circ}$ \\
\hline 1 & 0 & 0 & 1 & $90^{\circ}$ \\
\hline 1 & 0 & 1 & 0 & $180^{\circ}$ \\
\hline 0 & 1 & 1 & 0 & $270^{\circ}$ \\
\hline
\end{tabular}

After the character recognition process, the text is stored. We do the same thing to all the reference image plates one by one and store them in other text documents. When a car arrives at the garage gate, we compare two texts, one stored immediately after the car has approached and another, which was stored in the library previously. Block diagram of this process is depicted in Figure 9.

After comparing the two texts in MATLAB, we defined a variable, which will get logic 1 , and we will run a loop in the three motors to open the garage doors and pulley using smart controlling features [18, 19].

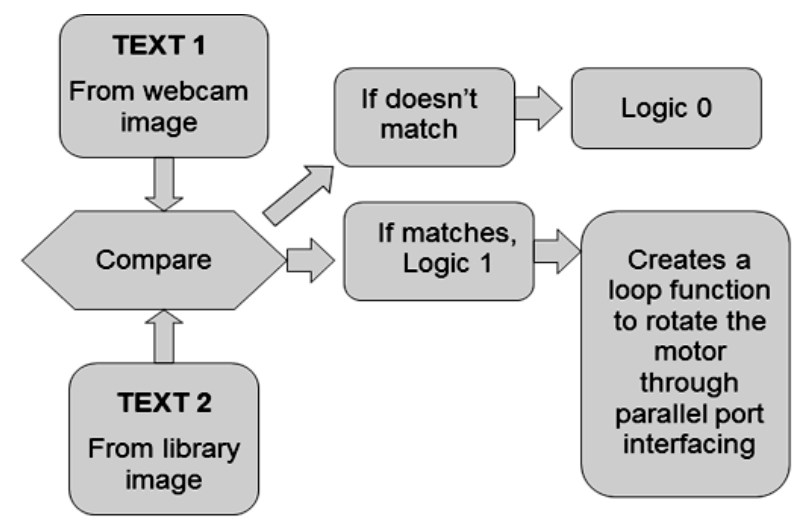

Fig. 9: Comparison of the license plate of an incoming vehicle to authorize the entrance in the garage

\section{Performance Analysis}

We have taken two pictures as a reference and stored in the library

\section{CTG253 \& DHK253}

\section{Observation 1:}

When we bring a car having a number plate of CTG 253 , it senses the number and passes the car by opening the gate. After it passes the second plate, the gates get closed.

The same result happens after we change the number plate to DHK 253. 


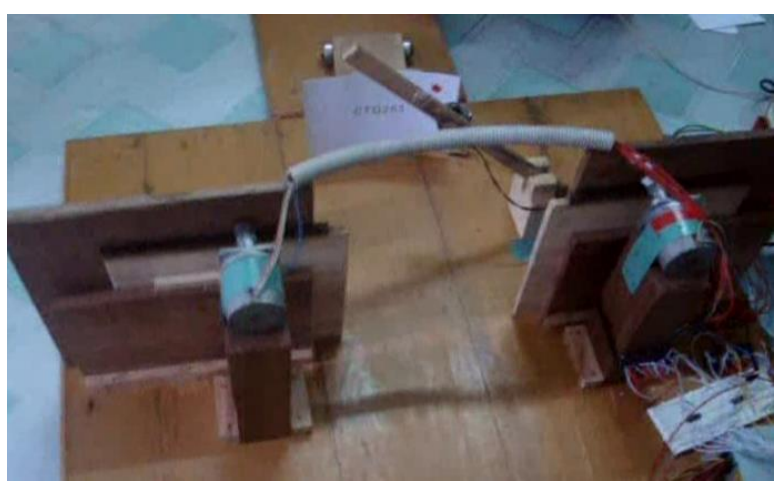

Fig. 10: Gate opens after recognizing the number plate

\section{Observation 2:}

Now we bring a car having a number plate of CTG 253

\section{CTG617}

It senses the number and glows a red light on the top of the cross bar

The same result happens after we change the number plate to DHK 617

\section{DHK617}

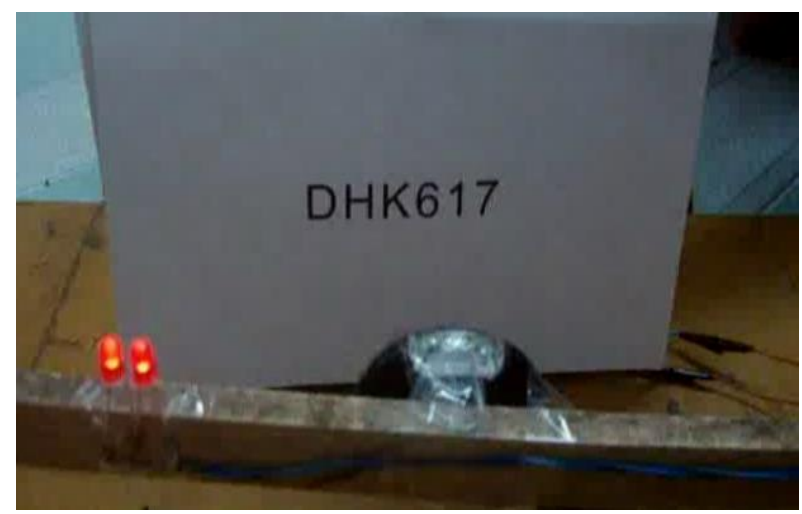

Fig. 11: Red light shows when the number plate is not recognized

\section{Features and Applications}

This is a very simple device to operate in any location with a cheaper cost. All the materials are available locally. As of the security purpose, it can be customized or modified to make the security level higher. As there is a weight sensor to activate the proceedings, the system does not work when only a license plate is shown to the camera. The system works even when the license plate is tilted a little bit. The practical application of the project is very wide. Some places where our project can be put to use, where specific cars are allowed to enter. For future work, we would like to improve recognition pattern in the physical dimension of the car. As such, the shape of the car can also be taken in the account for matching.
LVDT and IR sensor can be used instead of weight sensors to feel the presence of a vehicle. Furthermore, the gate can be made shutter type rather sliding one. This will save space and will be more suitable for domestic purposes. In addition, this system can also be used to keep records for the entire housing area of days and months so that the security purpose gets full filled.

\section{Conclusion}

The main aim of this project is to implement the LPR method to establish a secure garage system. Though we used gray-scale templates matching, hybrid method has a better performance in LPR. However, as the vehicle will have no motion during the snapshot. Thus, it is quite reliable. We can easily make a database of the cars that goes in and out in the garage. Furthermore, we can create a center alert system easily by using system. The pulley and the siding doors can be replaced by shuttle door that are used in personal garages. We can easily change the language of the LPR by just changing templates set. Alternatively, can use more than one language by just adding more language templates set in the templates archive. Considering our limitations, the purpose to design and analysis of an automated secure garage system with LPR can contribute in local garage system with affordable price and adjustable features. It can also contain a social security value.

\section{Acknowledgments}

The authors would like to thank Prof. Anil Kanti Dhar for his esteemed supervision. Also, express gratitude to the stuffs of CUET Workshop to assists in building the prototype.

\section{References}

[1] S. Yun, "A RKE Garage Door System Based on KEELOQ Technology [J]," Modern Electronics Technique, vol. 15, p. 48, 2008.

[2] D. C. Duhame, "Home security and garage door operator system," ed: Google Patents, 1984.

[3] D. C. Duhame, "Home security and garage door operator system," ed: Google Patents, 1982.

[4] T. Petersen, P. Williams, and A. Mills, "Analys is of the value of home automation systems," Facilities, vol. 19, pp. 522-532, 2001.

[5] R. D. Moss, "Security system for automatic door," ed: Google Patents, 2002.

[6] M. D. Abràmoff, P. J. Magalhães, and S. J. Ram, "Image processing with ImageJ," Biophotonics international, vol. 11, pp. 36-42, 2004. 
[7] Y. Zhu, J. Liu, X. An, J. Geng, and S. Chen, "A Design of One Kind Spring Layer Lift Intelligent Garage," in Dig ital Manufacturing and Automation (ICDMA), 2013 Fourth International Conference on, pp. 24-27, 2013.

[8] T.-H. Li, S.-J. Chang, and Y.-X. Chen, "Implementation of autonomous fuzzy garageparking control by an FPGA-based car-like mobile robot using infrared sensors," in Robotics and Automation, 2003. Proceedings. ICRA'03. IEEE International Conference on, pp. 3776-3781, 2003.

[9] L. Dongjing, "The achievement of Automatic Parking Control System of lifting and Transferring Stereo Garage based on real-time exchange parking stall," in Electric Information and Control Engineering (ICEICE), 2011 International Conference on, pp. 3657-3660, 2011.

[10] J. Shen, Y. Ye, Z. Zheng, and W. Hong, "Communication network design of stereo garage devices based on RS485," in Artificial Intelligence, Management Science and Electronic Commerce (AIMSEC), 2011 2nd International Conference on, pp. 3831-3834, 2011.

[11] J. Elliott, H. Jayachandran, P. Kumar, and K. Metzer, "Campus shuttle: Design of a college campus parking and transportation system," in Systems and Information Engineering Design Symposium (SIEDS), 2013 IEEE, pp. 104-109, 2013.

[12] P. Sánchez-Martín and G. Sanchez, "Optimal electric vehicles consumption management at parking garages," in PowerTech, 2011 IEEE Trondheim, pp. 1-7, 2011.

[13]J. Chinrungrueng, S. Du mn in, and R. Pongthornseri, "IParking: a parking management framework," in ITS Telecommunications (ITST), 2011 11th International Conference on, pp. 63-68, 2011.

[14] D. C. Hanselman, Mastering matlab 7: Pearson Education India, 2005.

[15] F. Kimura, T. Wakabayashi, S. Tsuruoka, and Y. Miyake, "Improvement of handwritten Japanese character recognition using weighted direction code histogram," Pattern recognition, vol. 30, pp. 1329-1337, 1997.

[16] W. Lee, D. Kim, and I. Kweon, "Automatic edge detection method for the mobile robot application," in Intelligent Robots and Systems, 2003.(IROS 2003). Proceedings. 2003 IEEE/RSJ International Conference on, pp. 2730-2735, 2003.

[17] M. Brown and S. Ganapathy, "Preprocessing techniques for cursive script word recognition," 1983.

[18] L. B. Rosenberg, A. C. Braun, and M. D. Levin, "Method and apparatus for controlling force feedback interface systems utilizing a host computer," ed: Google Patents, 1998.

[19] A. U. Ahmed, M. S. Khaled, M. Ali, and M. M. Rahman, "Controlling and Monitoring of Electric Feeders Using GSM Network Technology," Journal of Electrical and Electronics Engineering, vol. 6, pp. 5-8, 2013.

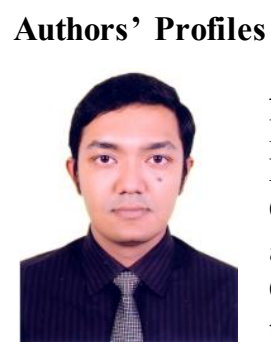

Afaz Uddin Ahmed obtained his B.Eng degree in Electrical and Electronic Engineering from Chittagong University of Engineering and Technology (CUET), Bangladesh. Currently, He is doing M.Eng.Sc. in the area of wireless communication in National University of Malaysia. He worked in Ericsson Bangladesh and Banglalink GSM (An ORASCOM Telecom Bangladesh Ltd.). His field of interest is LTE, heterogeneous network and next generation wireless communication system. $\mathrm{He}$ is a member of IEB (Institute of Engineers, Bangladesh) and Student member of IEEE.

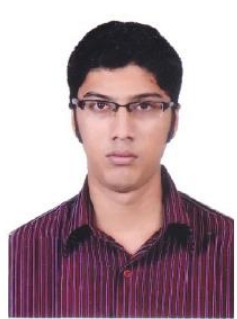

Taufiq Mahmud Masum had his B.Eng degree in Electrical and Electronic Engineering from CUET, Bangladesh. Currnetly he is working in Robi Axiata Limited as an system specialist. Previously he has worked for Ericsson Bangladesh. His field of interest is frequency and radio planning and resource allocation in heterogeneous network.

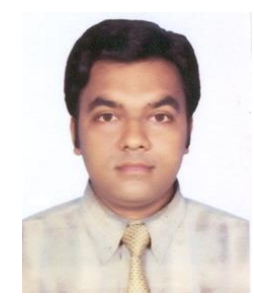

Mohammad Mahbubur Rahman had completed his $\mathrm{BSc}$ is in Electrical and Electronic Engineering from CUET, Bangladesh in 2010. He has been working as a Research assistant at the Department of Electrical Engineering in University of Malaya Malaysia since 2012. He also worked in Ericsson Bangladesh Ltd. His research interest includes OFDM, Electronic Communication, and Optical Fiber.

How to cite this paper: Afaz Uddin Ahmed, Taufiq Mahmud Masum, Mohammad Mahbubur Rahman,"Design of an Automated Secure Garage System Using License Plate Recognition Technique", International Journal of Intelligent Systems and Applications(IJISA), vol.6, no.2, pp.22-28, 2014. DOI: $10.5815 /$ ijisa.2014.02.03 